\title{
Modulation of tumor cell migration, invasion and cell-matrix adhesion by human monopolar spindle-one-binder 2
}

\author{
WENJUAN WU ${ }^{1,2^{*}}$, XIZHI ZHANG ${ }^{2 *}$, HAONAN QIN ${ }^{1}$, WANXIN PENG ${ }^{3}$, QINGYU XUE ${ }^{4}$, HOUNING LV $^{1}$,

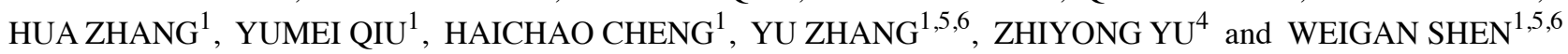 \\ ${ }^{1}$ School of Medicine and ${ }^{2}$ Clinical Medical College, Yangzhou University, Yangzhou, Jiangsu 225001; \\ ${ }^{3}$ School of Medicine, Jiangsu University, Zhenjiang, Jiangsu 212013; ${ }^{4}$ College of Biological and Chemical Engineering, \\ Yangzhou Vocational University, Yangzhou, Jiangsu 225000; ${ }^{5}$ Jiangsu Key Laboratory of Integrated Traditional Chinese and \\ Western Medicine for Prevention and Treatment of Senile Diseases, Yangzhou, Jiangsu 225001; ${ }^{6}$ Jiangsu Co-innovation Center \\ for Prevention and Control of Important Animal Infectious Diseases and Zoonoses, Yangzhou, Jiangsu 225009, P.R. China
}

Received November 22, 2014; Accepted February 27, 2015

DOI: 10.3892/or.2015.3855

\begin{abstract}
Human monopolar spindle-one-binder 2 (hMOB2) is a member of the hMOB family of proteins, and it has been reported to regulate the nuclear-Dbf2-related kinase (NDR) activation. However, the function of hMOB2 expression in tumor cell adhesion and motility has not been addressed. Herein, the lentiviral-mediated overexpression and the knockdown of hMOB2 in HepG2 and SMMC-7721 cells was established. It was demonstrated that overexpression of hMOB2 significantly reduced the cell motility and enhanced the cell-matrix adhesion, while the hMOB2 knockdown decreased not only the cell motility, but also the cell-matrix adhesion. Immunofluorescence results showed that both hMOB2 overexpression and knockdown altered assembly of the focal adhesions and the actin cytoskeleton rearrangement. Furthermore, the focal adhesion kinase (FAK)-Src-paxillin signal pathway activated by hMOB2 was confirmed to be involved in controlling the cell motility and the cell-matrix adhesion. These results demonstrated that the altered cellmatrix adhesion and cell motility induced by hMOB2 expression was caused by the assembly of focal adhesions as well as the actin cytoskeleton rearrangement through the activation of the FAK-Src-paxillin signal pathway, unveiling
\end{abstract}

Correspondence to: Professor Weigan Shen, School of Medicine, Yangzhou University, 11 Huaihai Road, Yangzhou, Jiangsu 225001, P.R. China

E-mail: shenweigan@hotmail.com

Professor Zhiyong Yu, College of Biological and Chemical Engineering, Yangzhou Vocational University, 458 Wenchang Road, Yangzhou, Jiangsu 225000, P.R. China

E-mail: jsyzyzy@163.com

*Contributed equally

Key words: hMOB2, cell motility, cell-matrix adhesion, focal adhesion a novel mechanism of cell motility and cell-matrix adhesion regulation induced by hMOB2 expression.

\section{Introduction}

Monopolar spindle-one-binder (MOB) family proteins are conserved from yeasts to human and function as the regulators of signaling pathways involved in cell mitosis, apoptosis and morphogenesis (1-9). Human MOB2 (hMOB2) is a member of the hMOB family of proteins, which contains at least 6 distinct hMOBs (hMOB1A, hMOB1B, hMOB2, hMOB3A, hMOB3B and hMOB3C) $(2,10)$. Among them, hMOB1A and hMOB1B have been characterized as the putative tumor suppressors in tumor cell proliferation, apoptosis and centrosome duplication through regulating the activation of the NDR kinase/large tumor suppressor kinase $(2,4-7,11)$, while the biological roles of other hMOBs are less well defined. To date, hMOB2 has also been widely termed hepatocellular carcinoma-associated gene 2 (HCCA2), and it is involved in the hepatocellular carcinoma development and progression (12). Nevertheless, data from the locus on the chromosome and the open reading frame (ORF) of mRNA of the hMOB2 gene (GenBank Accession: NM_053005, at position 11p15.5) and HCCA2 gene (GenBank Accession: AF206328, at position 1q22) revealed clear differences between them, suggesting that the biological behavior of hMOB2 may be different from the HCCA2. Therefore, it is currently unclear whether hMOB2 is involved in tumor development and progression.

In Drosophila, MOB2 plays a role in wing hair morphogenesis by interacting with the related tricornered and warts kinases $(13,14)$ and is involved in the photoreceptor cell development and rhabdomere formation by regulating the actin cytoskeleton rearrangement (15). The study also showed that mouse MOB2 promotes the neurite formation through regulating the actin cytoskeleton rearrangement (16). Currently, hMOB2 has been characterized to be involved in the regulation of hMOB1 by competing for the NDR1/2 kinases (8), which have already been linked to the actin cytoskeleton (17), although the mechanism involved is only partly understood. Since the dynamic remodeling of the actin cytoskeleton is 
also fundamental for many physiological processes like cell adhesion and cell motility (18), which contribute to tumor cell migration, invasion and metastasis (19), a better understanding of hMOB2-induced changes in the cytoskeletal regulation, cell adhesion and motility may result in better insight into tumor metastasis.

In the present study, we evaluated the effect of hMOB2 on tumor cell migration, invasion and cell-matrix adhesion. We found that hMOB2 overexpression appeared to significantly inhibit cell motility and promoted cell-matrix adhesion, while hMOB2 knockdown decreased not only the cell motility, but also the cell-matrix adhesion. Furthermore, we also demonstrated that both hMOB2 overexpression and knockdown altered assembly of the focal adhesions and the actin cytoskeleton rearrangement presumably through regulating the focal adhesion kinase (FAK)-Src-paxillin signaling pathway, unveiling a novel mechanism of cell motility and cell-matrix adhesion regulation induced by hMOB2.

\section{Materials and methods}

Cell lines and culture conditions. The human hepatocellular carcinoma cell lines HepG2 and SMMC-7721, and the human embryonic kidney (HEK) 293T cells were obtained from the Type Culture Collection of the Chinese Academy of Sciences (Shanghai, China), then cultured in Dulbecco's modified Eagle's medium (DMEM; Gibco) supplemented with 10\% heat-inactivated fetal bovine serum (FBS), $100 \mathrm{U} / \mathrm{ml}$ penicillin and $100 \mu \mathrm{g} / \mathrm{ml}$ streptomycin and maintained in a humidified incubator at $37^{\circ} \mathrm{C}$ with $5 \% \mathrm{CO}_{2}$.

Production of recombinant lentiviruses. To construct the lentiviral vector expressing hMOB2, the transfer vector pGC-hMOB2 was constructed by cloning the full-length of hMOB2 cDNA, amplified by polymerase chain reaction (PCR) from the hMOB2 cDNA clone which was obtained from the GeneChem Corporation (Shanghai, China) with the primers 5'-GAGGATCCCCGGGTACCGGTCGCCACCATGGACT GGCTCATGGGGAAG-3' (sense), and 5'-TCCTTGTAGTCC ATACCTCTCTCCTTCACGTGGTTCTG-3' (antisense), into the AgeI sites of pGV287 purchased from the GeneChem Corporation.

To construct the lentiviral vectors expressing siRNA targeting hMOB2, four different siRNA targeting sequences directed against hMOB2 were selected under the guide of siRNA designing software provided by GenScript. The sequences containing the hMOB2 siRNA targets (underlined sequence) were as follows: 5'-CCGGCATCACCGACTTCCA GTTCAACTCGAGTTGAACTGGAAGTCGGTGATGTTT TTG-3' (sense) and 5'-AATTCAAAAACATCACCGACTTC CAGTTCAACTCGAGTTGAACTGGAAGTCGGTGA-3' (antisense) for sihMOB2-1; 5'-CCGGCAGAGATTGACCTTA ACGAGTCTCGAGACTCGTTAAGGTCAATCTCTGTTTT TG-3' (sense), and 5'-AATTCAAAAACAGAGATTGACCTT AACGAGTCTCGAGACTCGTTAAGGTCAATCTC-3' (antisense), for sihMOB2-2; 5'-CCGGCATGTGCAACACACA GTACTACTCGAGTAGTACTGTGTGTTGCACATGTTTT TG-3' (sense), and 5'-AATTCAAAAACATGTGCAACACAC AGTACTACTCGAGTAGTACTGTGTGTTGCACA-3' (antisense), for sihMOB2-3; 5'-CCGGCAGCTGGTGACGGATG
AGGACCTCGAGGTCCTCATCCGTCACCAGCTGTTTT TG-3' (sense), and 5'-AATTCAAAAACAGCTGGTGACGG ATGAGGACCTCGAGGTCCTCATCCGTCACCAGC-3' (antisense), for sihMOB2-4. Then, the primers were synthesized and cloned into the AgeI and EcoRI sites of pGV115, purchased from the GeneChem Corporation.

For the lentiviral production, the lentiviruses encoding hMOB2 (LV-hMOB2) and siRNA against hMOB2 (LV-sihMOB2-1, LV-sihMOB2-2, LV-sihMOB2-3 and LV-sihMOB2-4) were produced by co-transfection of the lentiviral transfer vectors together with the packaging system pHelper 1.0 and 2.0 vectors purchased from the GeneChem Corporation into the HEK293T cells using Lipofectamine 2000 (Invitrogen, USA). The lentivirus particles were harvested and purified, and the infectious titers were determined by GeneChem Corporation. Control lentivirus (LV-CTL) and the non-silencing siRNA control lentivirus (LV-siCTL) were also generated by GeneChem Corporation and performed as the empty vectors for overexpression and knockdown, respectively.

For lentiviral infection, the HepG2 and SMMC-7721 cells were plated at a concentration of $1 \times 10^{5}$ cells in the 6 -well culture plates, and were then infected with indicated lentiviruses at a MOI of 20 and 30 in the presence of Polybrene $(8 \mu \mathrm{g} / \mathrm{ml})$, respectively. The infected cells continued to be cultured for over $96 \mathrm{~h}$ in DMEM supplemented with 10\% FBS. The green fluorescence protein (GFP), which was co-expressed in all the lentivirus-infected cells, served as a selection marker to indicate the successfully infected HepG2 or SMMC-7721 cells. To enrich the GFP-positive cells, the cells were sorted in the FACSCalibur (Becton-Dickinson), and the GFP-positive cells were returned to culture immediately, and were considered for the successful lentivirus-transduction in following experiments.

Cell migration and invasion assays. For the Transwell migration assay, the 24-well Transwell chambers with $8.0-\mu \mathrm{m}$ pore size of the porous membrane (Costar, USA) were performed. Briefly, the lentivirus-transduced cells were serum-starved for $24 \mathrm{~h}$ and then $3 \times 10^{4}$ cells in a volume of $200 \mu \mathrm{l}$ suspended in serum-free DMEM were seeded onto the upper chamber, which was placed into the lower chamber containing $600 \mu \mathrm{l}$ of DMEM supplemented with $10 \%$ FBS. After incubation for $24 \mathrm{~h}$ at $37^{\circ} \mathrm{C}$, the cells on the upper surface of the chambers were removed, and the cells that migrated to the lower surface of the filter were washed with phosphate-buffered saline (PBS), fixed with methanol for $15 \mathrm{~min}$ and stained with $0.1 \%$ crystal violet for $20 \mathrm{~min}$. The migrated cells in at least five randomly selected fields at x200 magnification were quantified, and images were captured using a phase contrast microscope equipped with a digital image capturing system. A cell invasion assay was also performed in the Transwell chambers as previously described with the minor modification that the upper chambers were precoated with $100 \mu \mathrm{g} / \mathrm{ml}$ Matrigel (BD Biosciences), and $200 \mu \mathrm{l}$ of $5 \times 10^{4}$ cells suspended in a serum-free DMEM were added to the upper chamber. The assays were performed in triplicate for each experiment and repeated three times.

Cell-matrix adhesion assay. For the cell-matrix adhesion assay, wells of 96-well culture plates were coated with $10 \mu \mathrm{g} / \mathrm{ml}$ collagen type I (BD Biosciences) overnight at $4^{\circ} \mathrm{C}$, blocked with $1 \%$ heat-denatured bovine serum albumin (BSA) 
in PBS at $37^{\circ} \mathrm{C}$ for $1 \mathrm{~h}$, and were then washed with PBS. The lentivirus-transduced HepG2 and SMMC-7721 cells were harvested, suspended at $5 \times 10^{5}$ cells $/ \mathrm{ml}$ in DMEM supplemented with $0.1 \%$ BSA and $100 \mu \mathrm{l}$ of the cell suspension were added to the coated well. After incubation at $37^{\circ} \mathrm{C}$ for $45 \mathrm{~min}$, non-adherent cells were gently removed by being washed four times with PBS. The numbers of the adherent cells were quantified by the Cell Counting Kit-8 (CCK-8; Obio Technology, Shanghai, China) assay according to the manufacturer's instruction followed by absorbance measurement at $450 \mathrm{~nm}$ using a multifunctional microplate reader (Bio-Tek, USA). The assays were performed in 6-wells and repeated five times.

$R N A$ preparation and real-time reverse transcription quantitative PCR (RT-qPCR). Total RNA was isolated from the lentivirus-transduced cells using the TRIzol reagent (Invitrogen), and reverse transcribed using the HiScript First Strand cDNA Synthesis kit (Vazyme, Nanjing, China) according to the manufacturer's instruction. RT-qPCR was used to determine the hMOB2 mRNA levels on an ABI 7500 Real-Time PCR System (Applied Biosystems, Carlsbad, CA, USA) using the AceQ ${ }^{\circledR}$ qPCR SYBR ${ }^{\circledR}$-Green Master Mix kit (Vazyme) according to the manufacturer's instruction. The primers for hMOB2 were: 5'-TTCCACCACATCAACCTGCA GTA-3' (sense), and 5'-GGAGCTCATGACGAAGTCAACG TA-3' (antisense); for GAPDH, 5'-GCACCGTCAAGGCTGA GAAC-3' (sense), and 5'-TGGTGAAGACGCCAGTGGA-3' (antisense), were used to run the RT-qPCR. The relative levels of the hMOB2 mRNA normalized to GAPDH mRNA were evaluated by $2^{-\Delta \Delta \mathrm{Ct}}$.

Western blot assay. The lentivirus-transduced cells were lysed in RIPA buffer (Beyotime, China) containing $1 \mu \mathrm{mol}$ phenylmethylsulfonyl fluoride and a protease and phosphatase inhibitor cocktail (Roche). After determination of the protein concentration with the Bradford method, the protein samples were electrophoresed by SDS-polyacrylamide gel electrophoresis (SDS-PAGE), and were then transferred onto the polyvinylidene fluoride membrane (PVDF, Millipore), which was subsequently blocked with $5 \%$ non-fat dried milk or 3\% BSA for tyrosine phosphorylation blots in TBS for $1 \mathrm{~h}$ at room temperature, and incubated with the indicated primary antibodies at $4^{\circ} \mathrm{C}$ overnight. The primary antibodies used in the present study were: polyclonal rabbit anti-hMOB2 (1:800; Abcam); monoclonal rabbit anti-FAK, polyclonal rabbit anti-pY397FAK, polyclonal rabbit anti-PY925FAK, monoclonal rabbit anti-Src, polyclonal rabbit anti-pY527Src (1:1,000; Cell Signaling Technology); monoclonal mouse anti-paxillin, polyclonal goat anti-pY118paxillin, monoclonal mouse anti-GAPDH (1:200; Santa Cruz Biotechnology). The PVDF membrane was then washed three times with TBS with $0.1 \%$ Tween-20 (TBST) followed by incubation for $2 \mathrm{~h}$ at room temperature with horseradish peroxidase-conjugated goat anti-rabbit IgG, goat anti-mouse IgG or donkey anti-goat IgG antibodies (1:2,000; KangChen Bio-tech, Shanghai, China). The protein bands were detected by enhanced chemiluminescence using the Pierce ECL Plus Western Blotting Substrate kit (Thermo Scientific).

Immunofluorescence analysis. The lentivirus-transduced cells were plated onto glass coverslips in 24-well culture plates and maintained in DMEM supplemented with 10\% FBS for $24 \mathrm{~h}$. The cells were washed with PBS, fixed with $4 \%$ paraformaldehyde diluted in PBS for $30 \mathrm{~min}$, permeabilized with $0.5 \%$ Triton X-100 in PBS for $10 \mathrm{~min}$, and then blocked with $3 \%$ BSA in PBS for $1 \mathrm{~h}$ at room temperature. After incubation overnight at $4^{\circ} \mathrm{C}$ with monoclonal mouse anti-paxillin antibody diluted 1:100 in blocking solution, the cells were washed three times with PBS and subsequently incubated with the Rhodamine-conjugated goat anti-mouse IgG (1:1,000; Biosource International) for $2 \mathrm{~h}$ at room temperature in the dark. Actin was visualized using Rhodamine-conjugated phalloidin (Sigma) at $37^{\circ} \mathrm{C}$ for $1 \mathrm{~h}$ at room temperature and then gently washed in PBS. All the cells were counterstained with DAPI (Sigma) for nucleus. After being washed thoroughly with PBS, the images were captured using a fluorescent microscope equipped with a digital image capturing system.

Statistical analysis. Data are expressed as the means \pm standard deviation (SD). Statistical significance was determined by the Student's t-test, and the p-values $<0.05$ were considered to indicate a statistically significant result.

\section{Results}

Expression of hMOB2 after lentiviral transduction. The overexpression and knockdown of hMOB2 in the lentivirus-transduced cells were initially examined. The levels of hMOB2 mRNA from the total RNA extracts were quantified using RT-qPCR, normalized to GAPDH mRNA and the hMOB2 protein levels from the whole cell extracts were determined by western blot assay. As shown in Figs. 1 and 2, overexpression of hMOB2 was observed in LV-hMOB2 transduced HepG2 and SMMC-772 cells both in mRNA (Fig. 1A) and protein levels (Fig. 1B and $\mathrm{C}$ ), while knockdown of hMOB2 expression by LV-sihMOB2-4 was achieved with the most efficient inhibition at the mRNA (Fig. 2A) and the protein levels (Fig. 2B and C) both in HepG2 and SMMC-772 cells. These results demonstrated that the indicated lentiviruses were successfully transduced into the HepG2 and SMMC-772 cells, and hMOB2 was overexpressed in LV-hMOB2-transduced, and the most efficient knockdown of hMOB2 was shown in the LV-sihMOB2-4-transduced cells. Therefore, LV-hMOB2 and LV-sihMOB2-4 (following named LV-sihMOB2) could be used in the following experiments.

Effect of hMOB2 on cell migration and invasion. To evaluate the role of hMOB2 in cell migration and invasion, we first performed a Transwell migration assay. As shown in Fig. 3, both HepG2 and SMMC-772 cells infected with LV-hMOB2 showed dramatic inhibition of the cell migratory ability, when compared with those transduced with the empty vectors (LV-CTL and LV-siCTL) and the mock. Notably, knockdown of the hMOB2 expression by LV-sihMOB2 also decreased the motile cells either in the HepG2 or the SMMC-772 cells (Fig. 3A-C). No significant differences in the cell migration were found among the mock and the empty vector-transduced cells. Similar effects of the hMOB2 overexpression and knockdown on cell invasion were also found in the Transwell cell invasion assay (Fig. 3D-F). Together, the results demonstrated that both overexpression and knockdown 
A
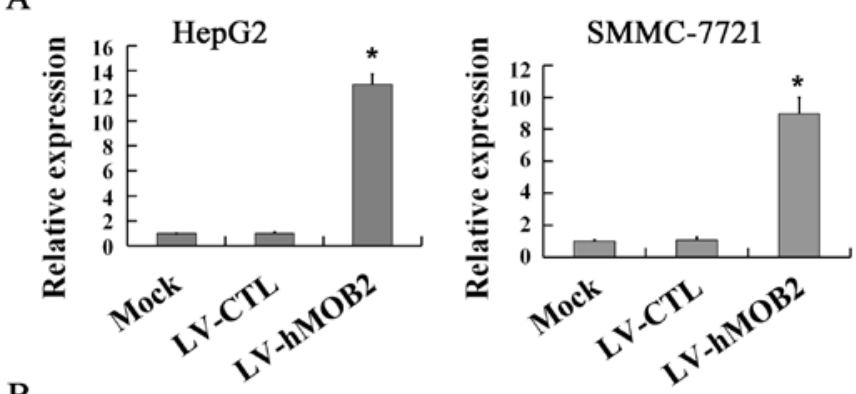

B
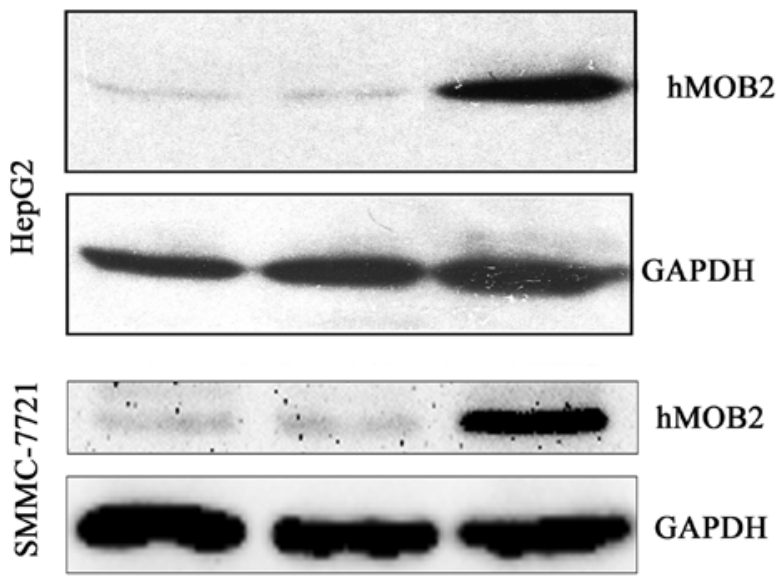

$\mathrm{C}$
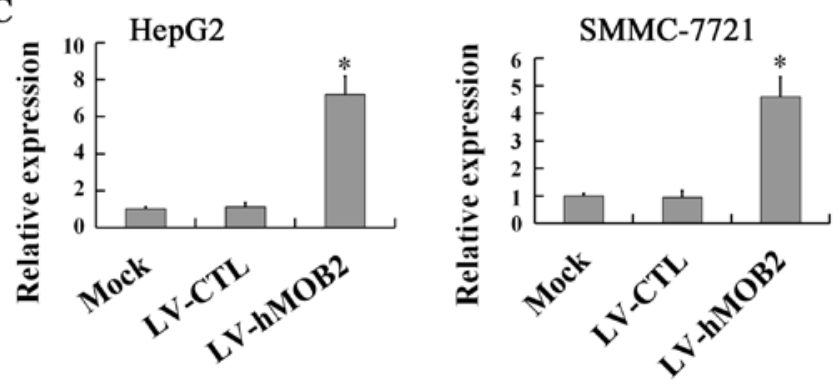

Figure 1. Overexpression of hMOB2 in the lentivirus-transduced cells. (A) hMOB2 mRNA levels were quantified in RNA extracts from the indicated lentivirus-transduced HepG2 and SMMC-7721 cells using RT-qPCR normalized against GAPDH. The values are the mean $\pm \mathrm{SD}(\mathrm{n}=5)$. ${ }^{*} \mathrm{p}<0.05$ vs. the mock. (B and C) The hMOB2 protein levels were determined by SDSPAGE immunoblotting with antibodies to hMOB2 and GAPDH, which was used as a loading control. The values are the mean $\pm \mathrm{SD}(\mathrm{n}=3)$. ${ }^{*} \mathrm{p}<0.05$ vs. the mock. hMOB 2, human monopolar spindle-one-binder 2 .

of hMOB2 attenuated the general capacity of cell migration and invasion.

Effect of $h M O B 2$ on the cell-matrix adhesion. Since cell migration and invasion require cell-extracellular matrix (ECM) adhesion and then detachment from ECM $(19,20)$, we next investigated the effect of hMOB2 on the cell-matrix adhesion. As shown in Fig. 4, hMOB2 overexpression obviously promoted both the HepG2 and SMMC-772 cell adhesion onto the collagen type I matrix, while hMOB2 knockdown decreased the adhesive behavior either in HepG2 or SMMC-772 cells, when compared with those in the empty vector-transduced cells and the mock. No significant difference in the cell-matrix adhesion was observed among the mock and the empty vector-transduced cells. Given the
A

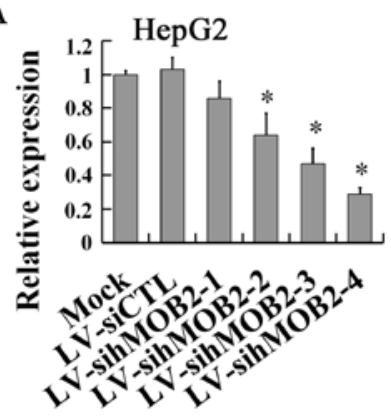

SMMC-7721

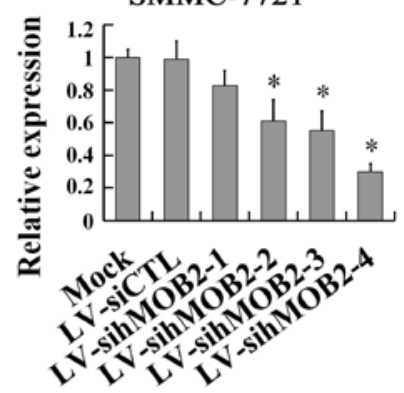

B
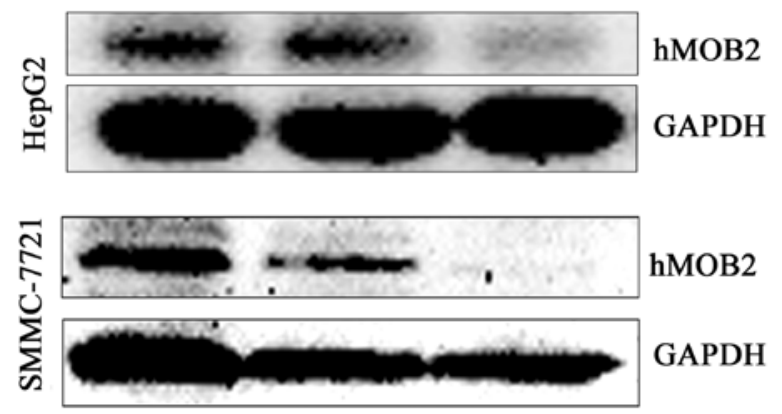

$\mathrm{C}$
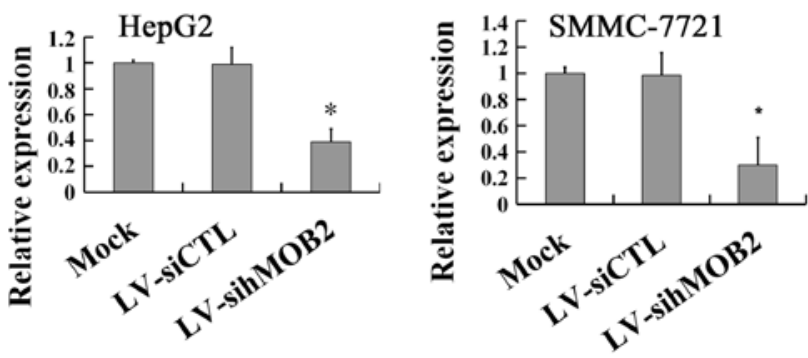

Figure 2. Knockdown of hMOB2 in the lentivirus-transduced cells. (A) hMOB2 mRNA levels were quantified from the indicated lentivirus-transduced HepG2 and SMMC-7721 cells using RT-qPCR normalized against GAPDH. The values are the mean $\pm \mathrm{SD}(\mathrm{n}=5)$. " $\mathrm{p}<0.05$ vs. the mock. (B and C) The hMOB2 protein levels were determined by SDS-PAGE immunoblotting with antibodies to hMOB2 and GAPDH, normalized against GAPDH. The values are the mean $\pm \mathrm{SD}(\mathrm{n}=3)$. ${ }^{*} \mathrm{p}<0.05$ vs. the mock. hMOB2, human monopolar spindle-one-binder 2.

results of the cell motility and the cell-matrix adhesion assays, reduction of the cell motility by hMOB2 overexpression may be the consequence of the strong or excessive cell-matrix adhesion, while the decrease in the cell motility caused by hMOB2 knockdown is most likely attributed to the decrease of the cell-matrix adhesion.

Effect of hMOB2 on the focal adhesion and the actin cytoskeleton rearrangement. The focal adhesions are cellular loci through which the actin filaments establish adhesive links between cell and ECM, and the dynamic assembly and disassembly of focal adhesion is essential for cell-matrix adhesion and cell motility (21-23). To further verify the implication of the hMOB2 expression in the cell-matrix adhesion and cell motility, we examined the focal adhesion formation by staining with paxillin (a marker of mature and nascent focal adhesion) and the remodeling of the actin cytoskeleton by staining with phalloidin in the lentivirus-transduced cells. In Fig. 5, hMOB2 overexpression significantly increased both the number and 


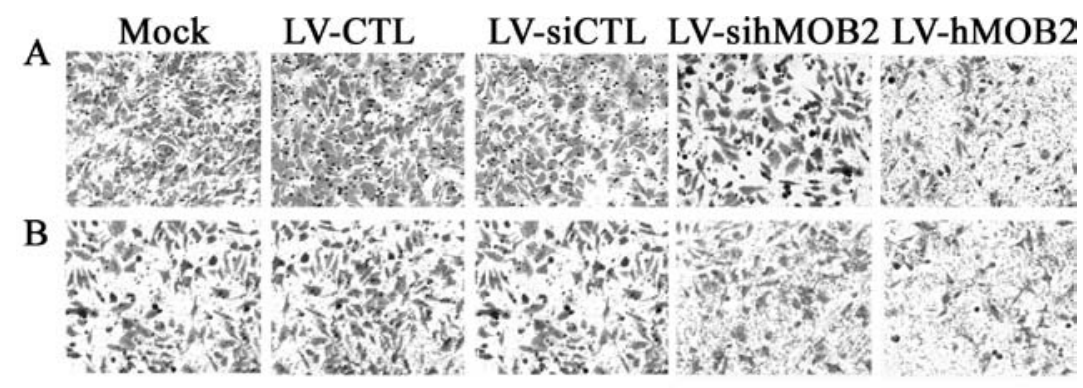

$\mathrm{C}$
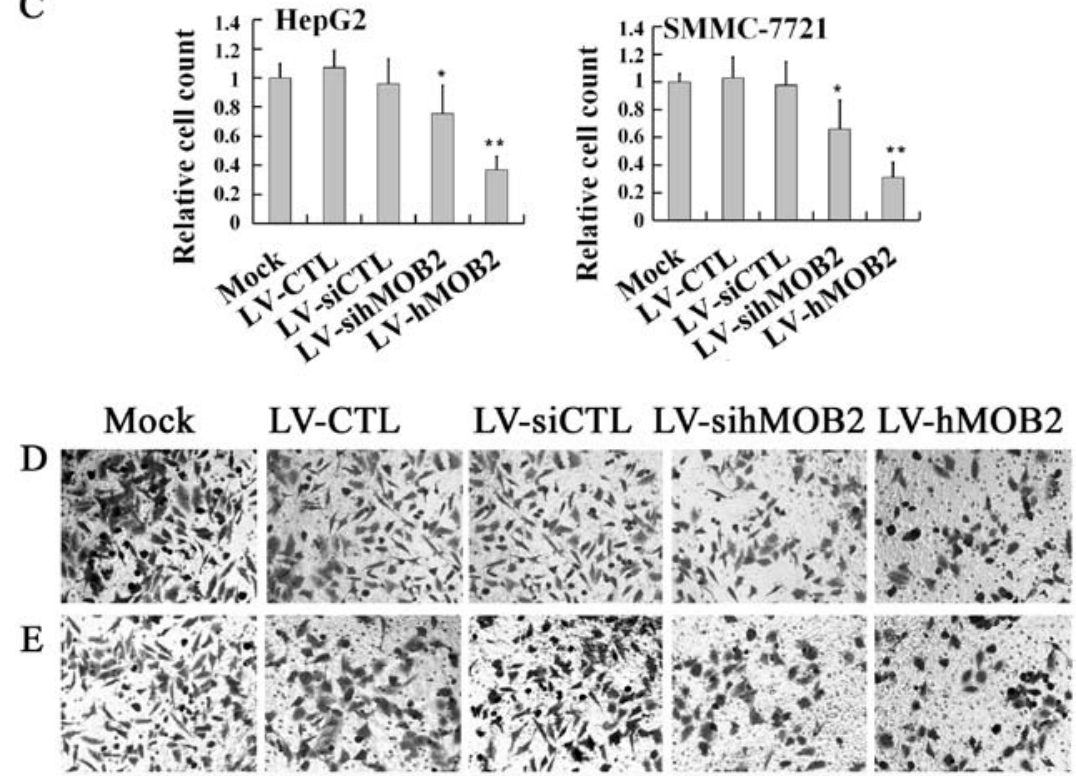

$\mathrm{F}$
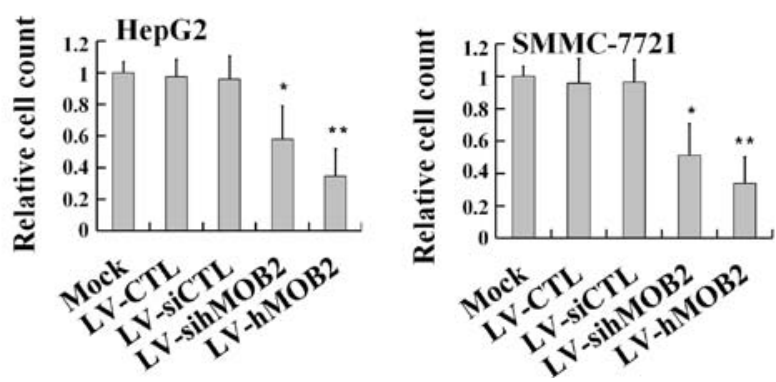

Figure 3. Effects of hMOB2 on cell migration and invasion. (A and B) The lentivirus-transduced (A) HepG2 and (B) SMMC-7721 cells were plated on the Transwell inserts and cell migration towards the low chambers containing DMEM supplemented with 10\% FBS and were recorded from at least five random images per insert from triplicate wells (magnification, x200). (C) Quantification of the migration of the lentivirus-transduced HepG2 and SMMC-7721 cells is shown. Assays were performed in triplicate from three independent experiments. The values are the mean $\pm \mathrm{SD}$. ${ }^{*} \mathrm{p}<0.05,{ }^{* *} \mathrm{p}<0.01 \mathrm{vs}$. the mock. (D and E) The lentivirus-transduced HepG2 and SMMC-7721 cells were plated on the Transwell inserts precoated with Matrigel and cell invasion was recorded from five random microscopic fields (magnification, x200) per insert from triplicate wells. (F) Quantification of the invasion of the lentivirus-transduced HepG2 and SMMC-7721 cells is shown. Assays were performed in triplicate from three independent experiments. The values are the mean \pm SD. ${ }^{*} p<0.05,{ }^{* *} p<0.01$ vs. the mock. hMOB2, human monopolar spindle-one-binder 2.
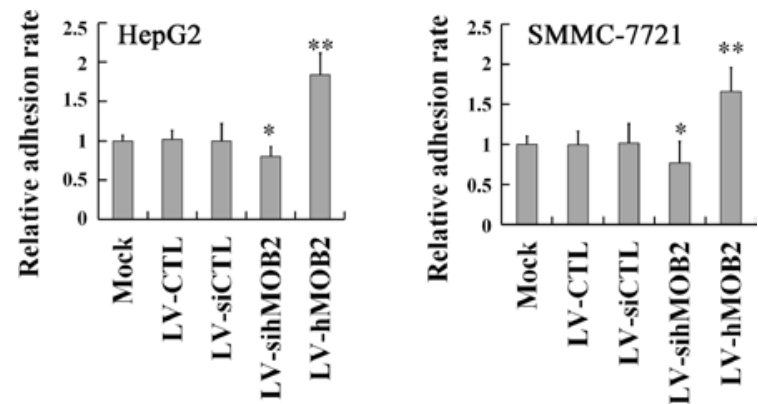

Figure 4. Effect of hMOB2 on cell-matrix adhesion. The lentivirus-transduced HepG2 and SMMC-7721 cells were seeded onto the collagen I precoated 96-well culture plates and incubated at $37^{\circ} \mathrm{C}$ for $45 \mathrm{~min}$. The number of the adhesive cells was quantified using the CCK- 8 assay by absorbance at $450 \mathrm{~nm}$. Assays were performed in 6-wells and repeated five times. The reported values are the mean $\pm \mathrm{SD}$. ${ }^{*} \mathrm{p}<0.05,{ }^{* *} \mathrm{p}<0.01 \mathrm{vs}$. the mock. hMOB2, human monopolar spindle-one-binder 2. 
A

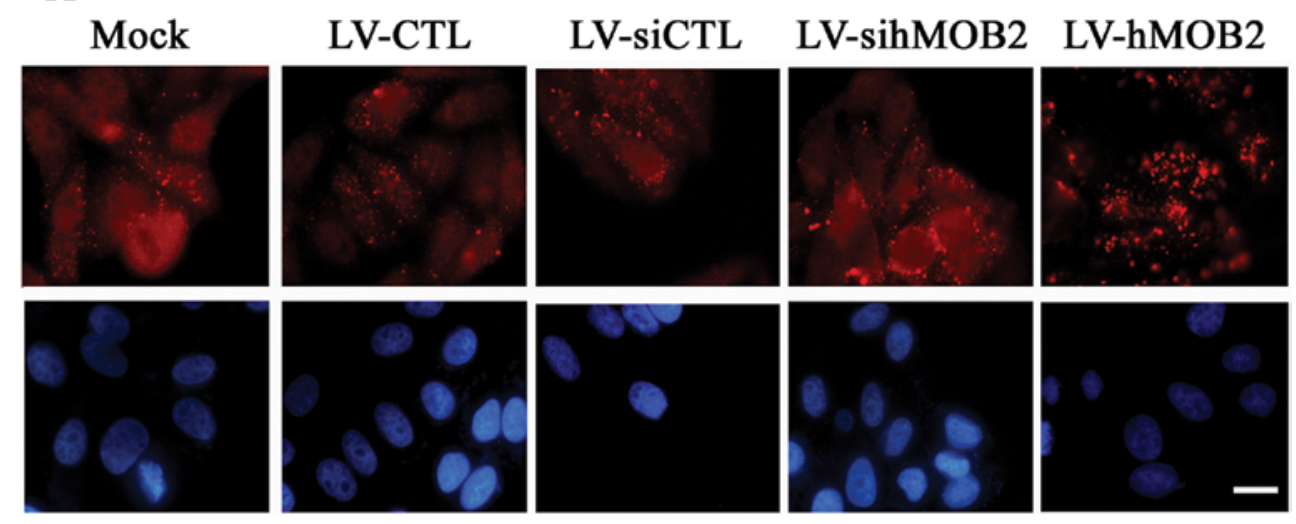

B

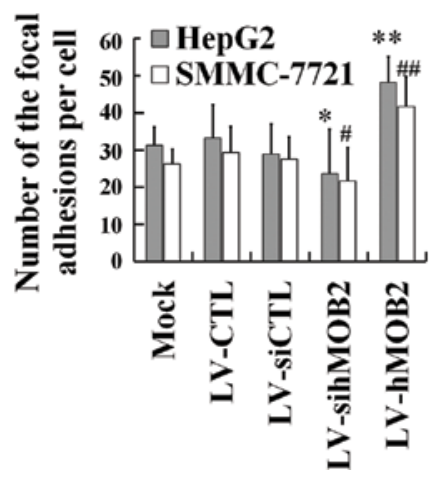

C

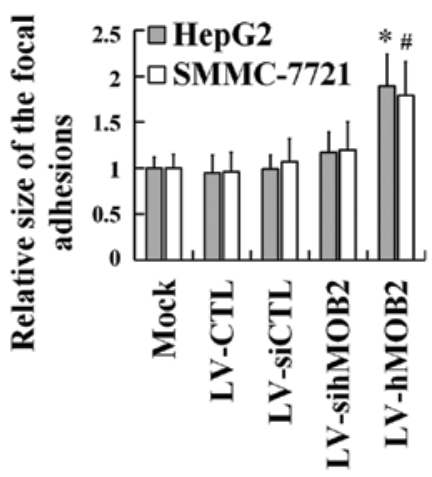

Figure 5. hMOB2 regulates the formation of focal adhesions. (A) Images show the immunofluorescence staining of paxillin (upper) accompanied by the nucleus (down) stained by DAPI in the HepG2 cells and represent the cells from three independent experiments. Scale bars, $20 \mu \mathrm{m}$. (B) Quantification of the number of focal adhesions per HepG2 and SMMC-7721 cells from at least 10 cells in each group is shown. The values are the mean \pm SD from three independent experiments. ${ }^{*} \mathrm{p}<0.05,{ }^{* *} \mathrm{p}<0.01$ vs. the HepG2 cell mock, and ${ }^{\#} \mathrm{p}<0.05,{ }^{\# \#} \mathrm{p}<0.01$, vs. the SMMC-7721 cell mock. (C) Quantification of the size of focal adhesions in HepG2 and SMMC-7721 cells from at least 80 focal adhesions in each group is shown, and three independent experiments were performed. The values are the mean $\pm \mathrm{SD} .{ }^{*} \mathrm{p}<0.05,{ }^{\#} \mathrm{p}<0.05$ vs. the indicated mock. hMOB 2 , human monopolar spindle-one-binder 2.
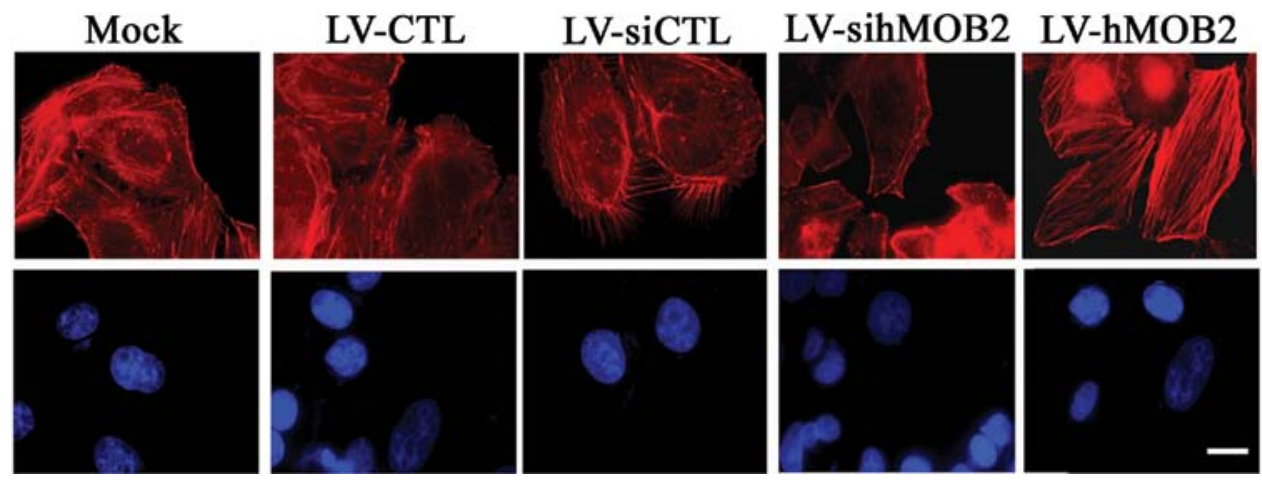

Figure 6. Effect of hMOB2 on the actin cytoskeleton rearrangement. The cells were fixed, permeabilized and stained with Rhodamine-conjugated phalloidin for F-actin (upper panel) and with DAPI for the nucleus (lower panel). Scale bars, $20 \mu \mathrm{m}$. The images represented the cells from three independent experiments. hMOB2, human monopolar spindle-one-binder 2.

the size of the focal adhesions and exhibited diffuse distribution of the focal adhesion throughout the HepG2 cells, while the hMOB2 knockdown reduced the number of the focal adhesions producing a prominent peripheral distribution of the HepG2 cells, when compared with those in the empty vector-transduced cells and the mock. In parallel, more stress fibers and less filopodia and lamellipodia at the leading edge were observed in the hMOB2-overexpressing HepG2 cells than those in the empty vector-transduced cells and the mock, whereas the hMOB2 knockdown resulted in the dissociation of the centrally located stress fibers and exhibited a peripheral distribution of the actin filaments (Fig. 6). No significant differences in the focal adhesion formation and the actin cytoskeleton rearrangement were observed among the mock and the empty vector-transduced cells. Similar results were also found in the SMMC-772 cells (data not shown). These data suggested that the altered assembly of the focal adhesions and the actin cytoskeleton rearrangement caused by hMOB2 overexpression and knockdown are possibly involved in the regulation of cell-matrix adhesion and cell motility. 
A

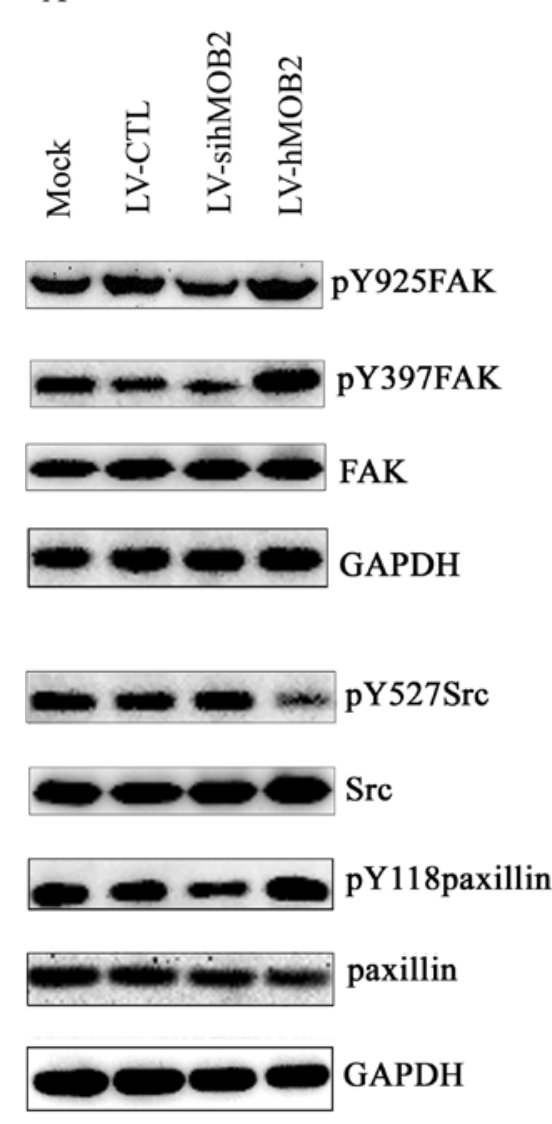

B
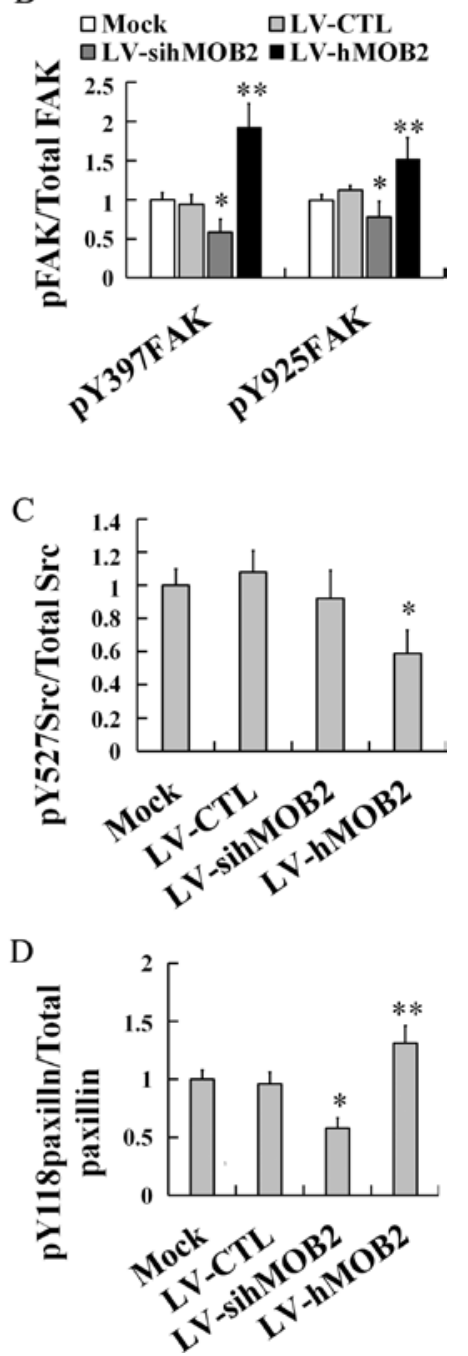

Figure 7. Effect of hMOB2 on the FAK-Src-paxillin signal pathway. (A) Whole HepG2 cell lysates were analyzed by SDS-PAGE immunoblotting with the indicated antibodies. GAPDH was used as a loading control $(n=3)$. (B-D) Quantification of the band intensity was performed with ImageJ software, and expressed as fold-change of the mock after being normalized with the GAPDH. The values are the mean \pm SD of three independent experiments. " $\mathrm{p}<0.05$, *** $<0.01$ vs. the mock. hMOB2, human monopolar spindle-one-binder 2.

Effect of hMOB2 on the FAK-Src-paxillin signaling pathway. Since cell-matrix adhesion and cell motility are dependent on the dynamic assembly and disassembly of focal adhesions and the remodeling of the actin cytoskeleton, which are regulated by a variety of signaling molecules, including FAK, Src and paxillin (24-26), we further performed western blot assay to evaluate the effects of hMOB2 on the expression of these signaling molecules. As shown in Fig. 7, hMOB2 overexpression upregulated the expression of phosporylation of FAK at Y397 (pY397FAK) and Y925 (pY925FAK), and phosporylation of paxillin at Y118 (pY118paxillin), and downregulated the phosphorylation of Src at Y527 (pY527Src) while hMOB2 knockdown obviously downregulated the expression of pY397FAK, pY925FAK and pY118paxillin, and resulted in only a slight but a non-significant reduction of the expression of the pY527Src, and no significant difference was found either in the LV-CTL-transduced or the LV-siCTL-transduced cells (data not shown). Neither hMOB2 overexpression nor hMOB2 knockdown had significant effects on the expression of the total FAK, Src and paxillin. Similar results were also found in the SMMC-772 cells (data not shown). Thus, these results suggest that FAK-Src-paxillin signaling pathway regulated by hMOB2 expression is likely involved in the regulation to the cell-matrix adhesion and cell motility.

\section{Discussion}

The findings presented here revealed a currently unknown role for hMOB2 in regulation of the cell-matrix adhesion and cell motility. The present study demonstrated that overexpression of hMOB2 significantly decreased the cell migration and invasion, but promoted the cell-matrix adhesion, while hMOB2 knockdown decreased not only the cell motility, but also the cell-matrix adhesion, revealing a novel regulatory mechanism of cell motility and cell-matrix adhesion induced by hMOB2 expression. Functional studies further disclosed an important role of hMOB2 in the modulation of the focal adhesion formation, the remodeling of the actin cytoskeleton and the FAK-Src-paxillin signaling. We demonstrated that the altered cell motility and cell-matrix adhesion induced by hMOB2 
expression was likely caused by its regulation to the assembly of focal adhesions and the actin cytoskeleton rearrangement through the activation of the FAK-Src-paxillin signal pathway.

Although hMOB2 has been reported to function as an inhibitor of NDR $1 / 2$ kinases in a binding-dependent manner (8), which has already been linked to the actin cytoskeleton (17), the function of hMOB2 expression in tumor cell migration and adhesion has not been addressed. Previous studies have demonstrated that mouse Mob2 and NDR activation in neuronal function have already been linked to the actin cytoskeleton rearrangement $(16,17)$, which is intimately associated with cell motility and adhesion. Therefore, our initial experiments were performed to investigate whether hMOB2 affects tumor cell migration and invasion. Our findings that hMOB2 overexpression obviously suppressed the cell migration and invasion and that hMOB2 knockdown also decreased the cell motility either in HepG2 or SMMC-772 cells suggests a mechanism by which overexpression and knockdown of hMOB2 attenuated the general capacity of cell motility. Since cell migration and invasion require cell-ECM adhesion and then release from ECM, decreased and strong or excessive cell-ECM adhesion will inhibit cell motility $(20,27)$. Next, it was evaluated whether hMOB2 overexpression and knockdown inhibited the cell motility through its regulation for the cell-matrix adhesion. Our findings that hMOB2 overexpression significantly promoted cell-matrix adhesion and that hMOB2 knockdown decreased cell-matrix adhesion raised the exciting possibility that the decrease in the cell motility by hMOB2 overexpression may be attributed to the strong or excessive cell-matrix adhesion, while the reduction in the cell motility caused by hMOB2 knockdown is most likely the consequence of the decrease of the cell-matrix adhesion.

Cell motility and cell-ECM adhesion depend on the dynamic assembly and disassembly of the focal adhesions $(20,22,23,28-30)$, which connect the bundles of actin filaments from cell to ECM and are often influenced by the formation of stress fibers, and the dynamic assembly of the actin cytoskeleton plays an essential role in cell migration and adhesion (18,31). It has also been well characterized that the strong stress fibers provide large focal adhesions, while weak stress fibers show small focal adhesions (31). Thus, we further performed in immunofluorescence assay to examine the effects of hMOB2 on the focal adhesions and the actin cytoskeleton rearrangement. We found that the increased number and size of focal adhesions which are diffusively distributed throughout the cell, and the robust stress fibers assembly as well as the decreased formation of filopodia and lamellipodia at the leading edge were exhibited in the hMOB2 overexpressing cells, suggesting that hMOB2 overexpression led to the induction of the number and the enlargement of the focal adhesions. This is consistent with the enhanced stress fiber formation and the reduction of the formation of filopodia and lamellipodia and exhibits the characteristics of the adhesive cells and makes sense with respect to the promoted the cell-matrix adhesion and subsequently suppressed the cell motility as mentioned above. The decreased number of the focal adhesions and the reduction of centrally located stress fiber assembly were observed in the hMOB2 knockdown cells in which a prominent peripheral distribution of the focal adhesions and the actin filaments was shown, which is consistent with the decrease of the cell-matrix adhesion and subsequently suppressed the cell motility. Therefore, our observations clearly supported the notion that the stress fiber formation is directly associated with the cell-matrix adhesion via the focal adhesions, while the formation of the filopodia and lamellipodia at the leading edge is associated with cell motility (32-34). Collectively, these results suggest that the modulation of cell motility and cell-matrix adhesion by hMOB2 overexpression and knockdown is possibly mediated through regulating the assembly of focal adhesions and the actin cytoskeleton reorganization, providing new insight into the function of hMOB2 in cell-matrix adhesion and cell motility.

It is generally understood for most cell types that the dynamic assembly and disassembly of the focal adhesion and the actin cytoskeleton are regulated by the FAK-Src signaling molecules (21-23), and the FAK-mediated focal adhesions linkage to the actin cytoskeleton were partially via binding to paxillin $(22,23,28,29)$. Upon activation of the FAK-Src signaling, autophosphorylation of FAK at Y397 creates a high-affinity binding site for Src and causes Src recruitment and activation, which thereafter phosphorylates other sites of FAK including Y925 resulting in the further activation of FAK, and other cellular scaffolding molecules including paxillin, thereby acting to modulate the focal adhesion dynamics and the actin cytoskeleton rearrangement during cell-matrix adhesion and motility $(23,24,31,35-40)$. Based on our findings that hMOB2 overexpression and knockdown regulated the cell motility and cell-matrix adhesion, we further evaluated the expression of some molecules involved in the FAK-Src signaling. We found that hMOB2 overexpression promoted autophosporylation of the FAK at Y397, downregulated the expression of pY527Src [the phosphorylation of Y527 inhibits Src activities (41)], and subsequently upregulated the expression of pY925FAK and pY118paxillin, whereas hMOB2 knockdown resulted in the downregulation of the expression of pY397FAK, pY925FAK and pY118paxillin, and a slight but non-significant reduction of the pY527Src. These data suggest that the altered cell-matrix adhesion and cell motility induced by hMOB2 overexpression and knockdown probably requires functional FAK-Src-paxillin signaling molecules to regulate the focal adhesion formation and the actin cytoskeleton rearrangement, although details need further study.

In summary, the data presented here demonstrated that hMOB2 modulated the cell motility and the cell-matrix adhesion as the result of the regulation of the focal adhesion formation as well as the actin cytoskeleton rearrangement through the activation of the FAK-Src-paxillin signal pathway. Although further study is required to address the underlying mechanisms as to how hMOB2 expression coordinates the FAK-Src-paxillin signal pathway, it is likely that hMOB2 modulates cell-matrix adhesion and motility, which will likely contribute to our insight into tumor metastasis and progression. These findings may reinforce a role for hMOB2 as a new molecular target for metastatic carcinoma.

\section{Acknowledgements}

The present study was supported in part by the Yangzhou Science and Technology Project (no. YZ2008095), the Science 
and Technology Innovation Fund Projects of Yangzhou University Student, the Yangzhou Vocational University Research Project (no. 07z10) and the National Nature Science Foundation of China (no. 81172278).

\section{References}

1. Hergovich A, Kohler RS, Schmitz D, Vichalkovski A, Cornils H and Hemmings BA: The MST1 and hMOB1 tumor suppressors control human centrosome duplication by regulating NDR kinase phosphorylation. Curr Biol 19: 1692-1702, 2009.

2. Hergovich A: MOB control: Reviewing a conserved family of kinase regulators. Cell Signal 23: 1433-1440, 2011.

3. Stavridi ES, Harris KG, Huyen Y, Bothos J, Verwoerd PM, Stayrook SE, Pavletich NP, Jeffrey PD and Luca FC: Crystal structure of a human Mob1 protein: Toward understanding Mob-regulated cell cycle pathways. Structure 11: 1163-1170, 2003.

4. Ponchon L, Dumas C, Kajava AV, Fesquet D and Padilla A: NMR solution structure of Mob1, a mitotic exit network protein and its interaction with an NDR kinase peptide. J Mol Biol 337: 167-182, 2004.

5. Lai ZC, Wei X, Shimizu T, Ramos E, Rohrbaugh M, Nikolaidis N, Ho LL and Li Y: Control of cell proliferation and apoptosis by mob as tumor suppressor, mats. Cell 120: 675-685, 2005.

6. Mrkobrada S, Boucher L, Ceccarelli DF, Tyers M and Sicheri F: Structural and functional analysis of Saccharomyces cerevisiae Mob1. J Mol Biol 362: 430-440, 2006.

7. Ho LL, Wei X, Shimizu T and Lai ZC: Mob as tumor suppressor is activated at the cell membrane to control tissue growth and organ size in Drosophila. Dev Biol 337: 274-283, 2010.

8. Kohler RS, Schmitz D, Cornils H, Hemmings BA and Hergovich A: Differential NDR/LATS interactions with the human MOB family reveal a negative role for human MOB2 in the regulation of human NDR kinases. Mol Cell Biol 30: 4507-4520, 2010.

9. Tavares A, Gonçalves J, Florindo C, Tavares AA and Soares H: Mob1: Defining cell polarity for proper cell division. J Cell Sci 125: 516-527, 2012.

10. Chow A, Hao Y and Yang X: Molecular characterization of human homologs of yeast MOB1. Int J Cancer 126: 2079-2089, 2010.

11. Florindo C, Perdigão J, Fesquet D, Schiebel E, Pines J and Tavares AA: Human Mob1 proteins are required for cytokinesis by controlling microtubule stability. J Cell Sci 125: 3085-3090, 2012.

12. Wang ZX, Wang HY and Wu MC: Identification and characterization of a novel human hepatocellular carcinoma-associated gene. Br J Cancer 85: 1162-1167, 2001.

13. He Y, Emoto K, Fang X, Ren N, Tian X, Jan YN and Adler PN: Drosophila Mob family proteins interact with the related tricornered (Trc) and warts (Wts) kinases. Mol Biol Cell 16: 4139-4152, 2005.

14. Emoto K, He Y, Ye B, Grueber WB, Adler PN, Jan LY and Jan YN: Control of dendritic branching and tiling by the Tricorneredkinase/Furry signaling pathway in Drosophila sensory neurons. Cell 119: 245-256, 2004.

15. Liu LY, Lin CH and Fan SS: Function of Drosophila mob2 in photoreceptor morphogenesis. Cell Tissue Res 338: 377-389, 2009.

16. Lin $\mathrm{CH}, \mathrm{Hsieh} \mathrm{M}$ and Fan SS: The promotion of neurite formation in Neuro2A cells by mouse Mob2 protein. FEBS Lett 585: 523-530, 2011.

17. Stork O, Zhdanov A, Kudersky A, Yoshikawa T, Obata K and Pape HC: Neuronal functions of the novel serine/threonine kinase Ndr2. J Biol Chem 279: 45773-45781, 2004.

18. Akakura S and Gelman IH: Pivotal role of AKAP12 in the regulation of cellular adhesion dynamics: Control of cytoskeletal architecture, cell migration, and mitogenic signaling. J Signal Transduct 2012: 529179, 2012.

19. Lock JG, Wehrle-Haller B and Strömblad S: Cell-matrix adhesion complexes: Master control machinery of cell migration. Semin Cancer Biol 18: 65-76, 2008.
20. Zong F, Fthenou E, Mundt F, Szatmári T, Kovalszky I, Szilák L, Brodin D, Tzanakakis G, Hjerpe A and Dobra K: Specific syndecan-1 domains regulate mesenchymal tumor cell adhesion, motility and migration. PLoS One 6: e14816, 2011.

21. Geiger B, Spatz JP and Bershadsky AD: Environmental sensing through focal adhesions. Nat Rev Mol Cell Biol 10: 21-33, 2009.

22. Lanning NJ, Su HW, Argetsinger LS and Carter-Su C: Identification of SH2B1 $\beta$ as a focal adhesion protein that regulates focal adhesion size and number. J Cell Sci 124: 3095-3105, 2011.

23. Webb DJ, Donais K, Whitmore LA, Thomas SM, Turner CE, Parsons JT and Horwitz AF: FAK-Src signalling through paxillin, ERK and MLCK regulates adhesion disassembly. Nat Cell Biol 6: 154-161, 2004.

24. Fraley SI, Feng Y, Krishnamurthy R, Kim DH, Celedon A, Longmore GD and Wirtz D: A distinctive role for focal adhesion proteins in three-dimensional cell motility. Nat Cell Biol 12: 598-604, 2010.

25. van Nimwegen MJ and van de Water B: Focal adhesion kinase: A potential target in cancer therapy. Biochem Pharmacol 73: 597-609, 2007.

26. Mitra SK and Schlaepfer DD: Integrin-regulated FAK-Src signaling in normal and cancer cells. Curr Opin Cell Biol 18: 516-523, 2006.

27. Ishikawa $\mathrm{T}$ and Kramer RH: Sdc1 negatively modulates carcinoma cell motility and invasion. Exp Cell Res 316: 951-965, 2010.

28. Geiger B, Bershadsky A, Pankov R and Yamada KM: Transmembrane crosstalk between the extracellular matrix - cytoskeleton crosstalk. Nat Rev Mol Cell Biol 2: 793-805, 2001.

29. Hynes RO: Integrins: Bidirectional, allosteric signaling machines. Cell 110: 673-687, 2002.

30. Gupton SL and Waterman-Storer CM: Spatiotemporal feedback between actomyosin and focal-adhesion systems optimizes rapid cell migration. Cell 125: 1361-1374, 2006.

31. Hotulainen P and Lappalainen P: Stress fibers are generated by two distinct actin assembly mechanisms in motile cells. J Cell Biol 173: 383-394, 2006.

32. Hall A: Rho GTPases and the actin cytoskeleton. Science 279: 509-514, 1998.

33. Ridley AJ: Rho GTPases and cell migration. J Cell Sci 114: 2713-2722, 2001.

34. Machacek M, Hodgson L, Welch C, Elliott H, Pertz O, Nalbant P, Abell A, Johnson GL, Hahn KM and Danuser G: Coordination of Rho GTPase activities during cell protrusion. Nature 461: 99-103, 2009.

35. Liu J, Di G, Wu CT, Hu X and Duan H: CEACAM1 inhibits cell-matrix adhesion and promotes cell migration through regulating the expression of N-cadherin. Biochem Biophys Res Commun 430: 598-603, 2013.

36. Luo M and Guan JL: Focal adhesion kinase: A prominent determinant in breast cancer initiation, progression and metastasis. Cancer Lett 289: 127-139, 2010.

37. Zhao J and Guan JL: Signal transduction by focal adhesion kinase in cancer. Cancer Metastasis Rev 28: 35-49, 2009.

38. Van Slambrouck S, Jenkins AR, Romero AE and Steelant WF: Reorganization of the integrin $\alpha 2$ subunit controls cell adhesion and cancer cell invasion in prostate cancer. Int J Oncol 34: 17171726,2009

39. Van Slambrouck S, Grijelmo C, De Wever O, Bruyneel E, Emami S, Gespach C and Steelant WF: Activation of the FAK-src molecular scaffolds and p130Cas-JNK signaling cascades by a1-integrins during colon cancer cell invasion. Int J Oncol 31: 1501-1508, 2007.

40. Crosara-Alberto DP, Inoue RY and Costa CR: FAK signalling mediates NF-kappaB activation by mechanical stress in cardiac myocytes. Clin Chim Acta 403: 81-86, 2009.

41. Bjorge JD, Jakymiw A and Fujita DJ: Selected glimpses into the activation and function of Src kinase. Oncogene 19: 5620-5635, 2000 . 